\title{
The new first-line defense: the potential of nasopharyngeal colonization in vaccine strategies
}

This article was published in the following Dove Press journal:

Vaccine: Development and Therapy

17 October 2016

Number of times this article has been viewed

\author{
Win-Yan Chan \\ Jonathan M Cohen \\ Jeremy S Brown \\ Centre for Inflammation and Tissue \\ Repair, UCL Respiratory, Division of \\ Medicine, University College London, \\ London, UK
}

\begin{abstract}
Pathogens that can colonize the upper respiratory tract include Streptococcus pneumoniae, Hemophilus influenzae, Neisseria meningitidis, Moraxella catarrhalis, and Staphylococcus aureus. While these pathogens commonly asymptomatically colonize the nasopharynx of healthy adults, disease progression may occur in some individuals. In addition to these respiratory pathogens, there are a large number of commensal species also found in the upper respiratory tract which only very rarely cause disease, creating a complex community of bacterial species in the nasopharynx. This review addresses the novel, potential strategies that utilize the interactions between both homologous and heterologous species in the nasopharynx to vaccinate individuals against pathogenic bacteria. These strategies include the mechanisms employed by colonizing bacteria to regulate the presence of other species in the nasopharynx and the effect that colonization of the nasopharynx has on the host immune response. Interventional strategies investigated so far include the introduction of nonpathogenic bacteria to the nasopharynx to immunize against a closely related species, controlled colonization using both wild-type and attenuated species, and the use of other nonpathogenic colonizers to express antigens from potential pathogens. All these approaches harness the ability of the colonization to induce a mucosal immune response that can protect against future infection. In this review, S. pneumoniae and $N$. meningitidis colonization are used as case studies for this approach as the immunological effects of colonization have been widely studied in animal and human models. Colonization-based strategies have great potential, and, in particular, the attenuated strain approach has produced some encouraging data in animal models. However, the strategy for attenuating virulence must be stringent and caused by highly stable mutations that are unlikely to revert. In addition, the consequences of artificial administration of genetically modified bacteria to the nasopharynx on the usual host microbiome are unknown and would need to be monitored carefully.
\end{abstract}

Keywords: Streptococcus pneumoniae, colonization, adaptive immunity, antibody, protein antigen, capsular antigen, Neisseria sp.

\section{Introduction}

\section{Nasopharyngeal commensal species}

The upper respiratory tract is a host to many commensal bacterial species that create a complex community of microbes. These commensal species include a number of potentially pathogenic bacteria that usually colonize the nasopharynx without further progression to disease, but occasionally can spread from the nasopharynx to the lungs or blood to cause serious infections such as pneumonia, septicemia, and meningitis. Potential pathogens found in the nasopharynx include Streptococcus pneumoniae,
Centre for Inflammation and Tissue Repair, UCL Respiratory, Division of Medicine, University College London, Rayne Building, 5 University Street, London WCIE 6JF, UK

Tel +442076796008

Fax +44 2076796973

Email jeremy.brown@ucl.ac.uk 
Hemophilus influenzae, Neisseria meningitidis, Moraxella catarrhalis, and Staphylococcus aureus. ${ }^{1}$ The nasopharynx provides a relatively stable environment in which the commensal flora can flourish and be transmitted to other hosts. ${ }^{2,3}$ The duration of carriage varies for each species and also between the different strains of the same species, from weeks to months and likely to be years for some species. The dynamics of nasopharyngeal colonization by different species are delicately balanced, and disturbances to this ecology may allow pathogenic organisms to cause disease. ${ }^{4}$ Factors that affect this balance include the acquisition of different species, interspecies interactions, bacterial interactions with the host, and interference by environmental factors. For instance, nasopharyngeal colonization by $S$. pneumoniae protects against $S$. aureus carriage, ${ }^{5}$ and conversely reduction in $S$. pneumoniae carriage by vaccination could lead to increased carriage of $S$. aureus. Similarly, in animal models, bacterial species compete in establishing colonization. ${ }^{6}$

Colonization of the nasopharynx by bacteria can influence the host immune system even in the absence of overt disease. Epidemiologic studies have shown that the development of asthma $^{7-9}$ and chronic obstructive pulmonary disease ${ }^{10,11}$ is related to the diversity of colonizing organisms in the nasal flora. In mice, lack of microbial colonization increases allergic airway inflammation, ${ }^{12,13}$ and colonization with multiple species protects against airway inflammation. ${ }^{14}$ Importantly, nasopharyngeal colonization by bacterial pathogens can be an immunizing event, stimulating both humoral and cellular adaptive immune responses that protect against either re-colonization or subsequent invasive disease. ${ }^{15-21}$ These observations suggest that novel vaccine strategies could harness the immunizing effects of nasopharyngeal colonization to prevent serious infections, and this is the subject addressed in this review with a particular focus on the potential of nasopharyngeal colonization for the prevention of S. pneumoniae and N. meningitidis infections, which are used as case studies that illustrate the benefits and potential drawbacks of this approach.

\section{Colonization and existing vaccines for S. pneumoniae and $\mathbf{N}$. meningitidis} S. pneumoniae

Colonization with $S$. pneumoniae is universal in the first few months of life, with between $50 \%$ and $90 \%$ of children aged under 2 years colonized at any one time, ${ }^{22-27}$ sometimes with multiple strains. ${ }^{28}$ Peak carriage rates occur at 3-5 years of age and then wane to $\sim 10 \%$ in adult life. ${ }^{6,29}$ Carriage prevalence depends on geographical location, and is generally higher in the developing world. ${ }^{30-32}$ While initial colonization events may persist for up to 4 months, duration appears to shorten with increasing age to $2-4$ weeks in adults. ${ }^{33}$ The proportion of $S$. pneumoniae colonization events associated with disease is low in healthy adults. However, as colonization is very common, $S$. pneumoniae is a leading cause of acute otitis media (OM), pneumonia, sepsis, and meningitis globally ${ }^{34-36}$ causing an estimated 2,858,000 severe pneumonia episodes and 411,000 deaths annually worldwide in infants. ${ }^{37}$ The use of vaccines targeting polysaccharide capsule antigen in children has reduced the overall incidence of pneumococcal disease. ${ }^{38,39}$ However, the adult vaccine fails to protect against pneumonia, ${ }^{40}$ a key cause of respiratory morbidity and mortality in elderly subjects with comorbidities. In addition, the existing vaccines have a high cost of manufacture and have major limitations in strain coverage, only protecting against between seven and 23 of the $93+S$. pneumoniae capsular serotypes. This restricted serotype coverage has led to the replacement of $S$. pneumoniae vaccine serotypes by non-vaccine serotypes as both colonizers of the nasopharynx and causes of disease. ${ }^{31,41-43}$ Hence, there is a strong interest in alternative vaccine strategies that target all $S$. pneumoniae strains and could also prevent lung infection.

In addition to the profound effect on the relative prevalence of vaccine and non-vaccine $S$. pneumoniae serotypes, the introduction of the pneumococcal conjugate vaccine to infant immunization schedules has also disrupted the ecology of the nasopharyngeal flora in general. ${ }^{44,45}$ Although the short- and long-term consequences of these changes are not yet clear, they still raise some potential concerns about the effects of vaccination. The complete eradication of all $S$. pneumoniae from the nasopharynx may remove the competition that $S$. pneumoniae exerts on other potentially pathogenic organisms, perhaps allowing their overgrowth. This could in turn lead to a greater incidence of disease caused by $H$. influenzae, $N$. meningitidis, S. aureus, or $M$. catarrhalis. ${ }^{1}$ This disruption to the nasal flora will likely have implications on the incidence of disease and antibiotic strategies in the future..$^{5,46}$

\section{N. meningitidis}

$N$. meningitidis is a pathogenic member of the Neisseria family, several of which are common human nasopharyngeal commensal species. ${ }^{47} N$. meningitidis is a major cause of rapidly progressive meningitis and septicemia, which, although rare, often result in death or permanent disability. Nevertheless, $N$. meningitidis is an asymptomatic nasopharyngeal colonizer in $\sim 10 \%$ of healthy individuals at any 
time. ${ }^{48,49}$ The carriage rate is the lowest in young children and highest in young adults, with the UK rates of $3 \%$ of children under 4 years, $24 \%-37 \%$ of the age group of $15-24$ years, and $<10 \%$ in older age groups. ${ }^{49,50}$ Similar to $S$. pneumoniae, carriage rates are higher in smokers and after viral respiratory tract infections. ${ }^{48,51}$

Twelve different meningococcal serogroups have been defined based on capsular polysaccharide structure, of which A, B, C, W135, and Y are responsible for the majority of the disease. In addition, serogroup $\mathrm{X}$ has more recently been identified as the cause of sepsis and meningitis in Africa. ${ }^{52}$ Group A causes large-scale epidemics mainly in Africa but also in Asia, whereas the majority of cases of N. meningitidis disease in Europe and America are caused by serogroups B and C strains. As with $S$. pneumoniae, vaccines are currently polysaccharide based with newer vaccines utilizing protein conjugation to offer improved protection in children. In the $\mathrm{UK}$, a conjugate vaccine to protect against serotype $\mathrm{C}$ has been in use since 1999 in infants, and also for teenagers and young adults. Unlike the serotype replacement seen with the introduction of the pneumococcal conjugate vaccine, the introduction of a vaccine for meningococcus $\mathrm{C}$ has not seen a rise in carriage or disease by meningococcus $\mathrm{B}$. A conjugate vaccine targeting groups A, C, W135, and Y has been administered to adolescents since early 2016. Serogroup B has a capsule that is particularly poorly immunogenic, and the meningococcus B vaccine is based on outer membrane vesicles and protein antigens rather than capsular polysaccharide. This vaccine was added to the UK infant immunization schedule in 2015. Vaccine-related reduction in carriage may have contributed to herd immunity as was seen for S. pneumoniae. ${ }^{53,54}$

\section{Regulation by commensal species}

The nasal flora is acquired shortly after birth and is influenced by the environment, including contact with other persons. ${ }^{55}$ The competition between potentially pathogenic bacteria and commensal species in the nasopharynx contributes to the regulation of pathogenic species, which are also influenced by host responses. Environmental changes such as the season will influence the makeup of the nasal flora, ${ }^{56}$ as will therapeutic interventions such as vaccination or antimicrobials. For example, children with pneumococcal OM treated with antibiotics, or those who were immunized with the pneumococcal conjugate vaccine, had a decrease in the prevalence of Streptococcaceae and Corynebacteriaceae commensal species in the nasopharynx. ${ }^{57}$ Nasopharyngeal commensal species could prevent respiratory and invasive disease caused by pathogenic commensal species through a number of different mechanisms. These include the inhibition of colonization by potential pathogens by competition, either passively (occupying the same ecological niche) or actively (via direct growth inhibition or killing of competitor species). For example, in the gut, competition for nutrients causes a process referred to as colonization resistance, which is integral to controlling pathogenic bacteria such as enterohemorrhagic Escherichia coli, or Clostridium difficile, ${ }^{58,59}$ and similar processes are likely in the nasopharynx. Commensal species can also produce antimicrobial peptides that directly affect pathogen growth or survival. For example, the poorly pathogenic commensal species, Streptococcus salivarius, produces bacteriocins that inhibit $S$. pneumoniae, ${ }^{60,61}$ and in the gut enterohemorrhagic E. coli is inhibited by bacteriocins produced by other E. coli strains. ${ }^{62}$ On the skin, the commensal species, Staphylococcus epidermidis, produces antimicrobial proteins that prevent $S$. aureus growth. ${ }^{63}$ Another mechanism that directly inhibits other bacteria species is the production of hydrogen peroxide $\left(\mathrm{H}_{2} \mathrm{O}_{2}\right)$. S. pneumoniae is remarkably tolerant to $\mathrm{H}_{2} \mathrm{O}_{2}$ and although potential pathogens such as $S$. aureus and $H$. influenzae produce a catalase to neutralize $\mathrm{H}_{2} \mathrm{O}_{2}$, the concentrations produced by $\mathrm{S}$. pneumoniae overwhelm these catalases without killing the $S$. pneumoniae itself. ${ }^{46,64}$ Another strategy employed by $S$. pneumoniae is the production of neuraminidase, an enzyme that degrades $H$. influenzae cell-surface sialic acids, impairing the ability of $H$. influenzae to colonize the host. ${ }^{65}$ Commensal nasopharyngeal flora can inhibit the growth of group A Streptococcus, although the mechanisms are not clear. ${ }^{66}$ Finally, the impact of nasopharyngeal bacteria and viruses on other species can also be mediated via the modulation of the host's immune response. ${ }^{4}$ For example, in a mouse model, initial colonization with $H$. influenzae stimulated an innate immune response via immune recognition of cell wall components that enhanced phagocytosis of S. pneumoniae and inhibited colonization. ${ }^{67}$

\section{Immunizing effect of colonization}

Both human and animal data demonstrate that colonization is an immunizing event that prevents subsequent $S$. pneumoniae infection by both homologous and heterologous strains. Antibodies targeting capsular polysaccharides are detected in the serum of children following colonization, ${ }^{68}$ although the strength of the immune response depends on the infecting serotype. ${ }^{69,70}$ Exposure to a greater number of serotypes also enhances immune responses. ${ }^{71}$ In addition to anti-capsular antibodies, colonization in humans and in 
animal models induces antibodies to surface and intracellular S. pneumoniae protein antigens, many of which are protective. ${ }^{6,72}$ Unlike anti-capsular responses, anti-protein responses are rapidly detectable in the first year of life. ${ }^{68,73,74}$ However, an epidemiological study in infants did not find evidence that anti-protein antibodies protected against subsequent colonization. ${ }^{75}$ Colonization of adult volunteers with serotype $23 \mathrm{~F}$ or $6 \mathrm{~B}$ strains of $S$. pneumoniae led to the production of serum IgG to the protein antigens PspC and PspA and salivary $\operatorname{IgA}$ to $\mathrm{PspA}^{76}$ and preexisting serum anti-PspA IgG levels correlating with protection against experimental colonization. ${ }^{77}$ In another experimental human challenge model, nasopharyngeal colonization with a serotype $6 \mathrm{~B}$ strain of $S$. pneumoniae was established in healthy adults. Rechallenge failed to result in a second carriage event by the same strain, with protection persisting for up to 1 year. ${ }^{21}$ In these challenge studies, colonization resulted in cellular and humoral immune responses to $S$ pneumoniae. Data from mouse colonization models indicate that nasopharyngeal colonization leads to Th17-cell responses ${ }^{16,18,19}$ that enhance phagocyte recruitment to the nasopharynx, ${ }^{18,78}$ and are critical for both the initial clearance of the colonizing strain and subsequent protection against recolonization. The impact of these phagocytic responses is enhanced by the effects of specific antibody via opsonization and agglutination of $S$. pneumoniae. ${ }^{79}$ A murine model of group A Streptococcus nasopharyngeal infection has also been established. ${ }^{80}$ In this model, rapid clearance of recolonization was also dependent on an antigen-specific Th17-cell response. ${ }^{81}$ These data raise the possibility that Th17-cell mechanisms may be broadly important in the control of bacterial colonization of the nasopharynx.

Human colonization also leads to serum antibody responses that are able to protect against sepsis caused by heterologous strains in mouse challenge models, ${ }^{76}$ indicating that colonization-induced anti-protein responses are sufficient to enable protection. In addition to Th17-cell responses, the antibody response to colonization is also important for protection against $S$. pneumoniae lung infection in a mouse model. ${ }^{19}$ In the human experimental challenge model, colonization augmented $S$. pneumoniae-specific IL-17-secreting CD4+ T-cells in the human lung, ${ }^{20}$ suggesting that there may also be a role for colonization-induced Th17 cells in limiting lung infection in humans.

Overall, these observations demonstrate that colonization of healthy humans induces a mucosal and systemic immune response that protects against further colonization (in humans) or sepsis (in mice), and support data from animal models show similar findings. ${ }^{15,16,18,19}$ Importantly, intranasal administration of the $6 \mathrm{~B}$ strain without successful colonization also augmented local mucosal serological responses ${ }^{82}$ showing that exposure of the nasopharynx to the organism can stimulate an immunological response even if colonization was not detected.

Colonization of the upper airways with $N$. meningitidis is also an immunizing event that induces an antibody response persisting several weeks following acquisition. ${ }^{83,84}$ Interestingly, childhood nasopharyngeal exposure to commensal strains of Neisseria increases antibody levels which is largely strain-specific but has some degree of cross-reactivity with $N$. meningitidis, and which perseveres for several months. ${ }^{85-87}$

Overall, there is now an abundance of data indicating that nasopharyngeal colonization with potentially pathogenic bacteria elicits both humoral and cellular protective adaptive immune responses in humans and mouse models. These data support colonization as a novel alternative vaccine strategy to induce protection against bacterial pathogens. ${ }^{88,89}$

\section{Colonization as a vaccine strategy}

There are two potential strategies by which colonization could be used to prevent disease. The first is through harnessing the regulatory effects of commensal species on colonization by potential pathogens through competition for resources, immune modulatory effects, the secretion of bacteriocins, or other direct inhibitory mechanisms. The second is by stimulating a protective adaptive immune response, which unlike the first strategy requires colonization by an organism with significant antigenic overlap to the target pathogen.

\section{Prevention of colonization using commensal species}

The delivery of nonpathogenic commensal species as "probiotics" has been investigated for the prevention of OM. In some cases, the impact on bacterial nasopharyngeal colonization has also been assessed..$^{90}$ These avirulent organisms were administered orally or via nasal spray, and the preliminary results suggest that they can reduce the incidence of upper respiratory infections. ${ }^{91}$ In a study of adults given an oral mixture of organisms (containing Lactobacillus rhamnosus GG, Bifidobacterium, Lactobacillus acidophilus, and Streptococcus thermophilus), there was a significant reduction in nasal colonization with potential pathogens, including $S$. aureus, $S$. pneumoniae, and $\beta$-hemolytic streptococci. ${ }^{92}$ Studies in children given milk supplemented with Lactobacillus rhamnosus GG tend to show a reduction in respiratory infections, including OM, but have produced mixed results in the impact on the carriage of pathogens such as S. pneumoniae 
and H. influenzae. ${ }^{93,94}$ These studies delivered the commensal organisms to the gut where they might enhance general mucosal immunity through interactions with the gut-associated immune system..$^{92}$

Children who suffer from repeated episodes of OM are less likely to carry the oropharyngeal commensal alphahemolytic streptococci (AHS), whereas H. influenzae is more prevalent. ${ }^{95}$ This observation perhaps suggests that AHS have inhibitory effect on $H$. influenzae growth, similar to the ability of Streptococcus oralis to inhibit $S$. pneumoniae growth. ${ }^{96}$ This potential effect has been exploited by the oral administration of another AHS species $S$. salivarius, which was associated with a reduction in $S$. pyogenes infections in humans ${ }^{97,98}$ and inhibition of $S$. pneumoniae infection in mouse models..$^{99,100}$ Furthermore, nasal spray administration of five strains of AHS from three species (Streptococcus sanguis, Streptococcus mitis, and $S$. oralis) reduced the incidence of recurrent OM and secretory OM in children. ${ }^{101}$ However, in another study, there was no significant change in the incidence of OM although there was a trend toward reduced carriage of $H$. influenzae. ${ }^{102}$ It is possible that in these studies, the administration of antibiotics prior to the bacterial nasal spray may have enabled stable colonization with AHS strains and therefore may make a positive result more likely.

\section{Colonization to induce adaptive immunity}

The remainder of this review focuses on using intranasal administration of bacteria as an immunizing event; these approaches are summarized in Table 1. This approach has a number of potential advantages to using conventional vaccines. A whole bacterial cell approach means that the immunological response is not restricted to selected antigens and will induce redundant responses to multiple antigens, improving cross-reactivity between strains and reducing the potential for naturally occurring vaccine escape mutations. In addition, the vaccine would be inexpensive to manufacture and would not need an adjuvant as antigens are presented in an immunostimulatory context of the whole bacterium. Nasal administration also offers the advantages over parenteral administration of higher safety levels, needleless delivery, and improved immunity at the mucosal surface which may be more likely to prevent respiratory tract infections. The key principles of colonization-induced immunity (Figure 1) are detailed in the following sections.

\section{Cross-reactive protection between commensal and potentially pathogenic species}

Colonization with a commensal species could potentially enhance the clearance of a closely related potential pathogen if there are shared antigens between the species. This has been explored for the closely related species Neisseria lactamica and $N$. meningitidis. $N$. lactamica expresses antigens similar to those expressed by $N$. meningitidis, and sera from mice immunized with $N$. lactamica enhance $N$. meningitidis killing, ${ }^{103}$ and human carriage of $N$. lactamica results in a high titer of antibodies to $N$. meningitidis. ${ }^{104}$ Outer membrane proteins and lipooligosaccharide structures common to both species are the major antigenic sources of crossprotection. ${ }^{105} \mathrm{~N}$. lactamica colonization has been studied as a vaccination strategy to prevent $N$. meningitidis disease. In one study of colonization of healthy volunteers, the mucosal and systemic antibody response against $N$. lactamica was cross-reactive against $N$. meningitidis. However, while these antibodies were opsonophagocytic in vitro, they had poor

Table I Examples and potential mechanisms for inducing adaptive immunity to bacterial pathogens by nasopharyngeal colonization with live bacteria

\begin{tabular}{|c|c|c|c|c|}
\hline Type of approach & Target pathogen & Species/mutation(s) & Description & References \\
\hline $\begin{array}{l}\text { Commensal } \\
\text { cross-reactivity }\end{array}$ & N. meningitidis & N. lactamica & Induction of cross-reactive antibody & 103,104 \\
\hline Attenuated & S. pneumoniae & $c p s$, ply, and pspA & Virulence factor deletion & 107 \\
\hline \multirow[t]{7}{*}{ pathogenic bacteria } & S. pneumoniae & cps, teichoic acids, ply & Virulence factor deletion & 108,109 \\
\hline & S. pneumoniae & pep27 & Capsule reduction & 110 \\
\hline & S. pneumoniae & $f t s Y, \operatorname{caxP} / m g t A$ & Metabolic component deletion & 111 \\
\hline & S. pneumoniae & pabB & Auxotroph & 112 \\
\hline & S. pyogenes & spe $B$ and gid $A$ mutation & Impaired tRNA modification & 113 \\
\hline & Salmonella enterica serovar & gidA mutation & Impaired tRNA modification & 114 \\
\hline & Typhimurium & & & \\
\hline Heterologous & S. pneumoniae & PspA expression by Lactobacillus casei & Protective antigen expression & 118 \\
\hline \multirow[t]{2}{*}{ antigen expression } & S. pneumoniae & $\begin{array}{l}\text { PspA, PpmA, PsaA, PppA, and SIrA } \\
\text { expression by L. lactis }\end{array}$ & Protective antigen expression & 119 \\
\hline & S. pneumoniae & Cps expression by L. lactis & Protective antigen expression & $120,12 \mid$ \\
\hline
\end{tabular}

Abbreviations: L. lactis, Lactococcus lactis; N. lactamica, Neisseria lactamica; N. meningitidis, Neisseria meningitidis; S. pneumoniae, Streptococcus pneumoniae; S. pyogenes, Streptococcus pyogenes.. 


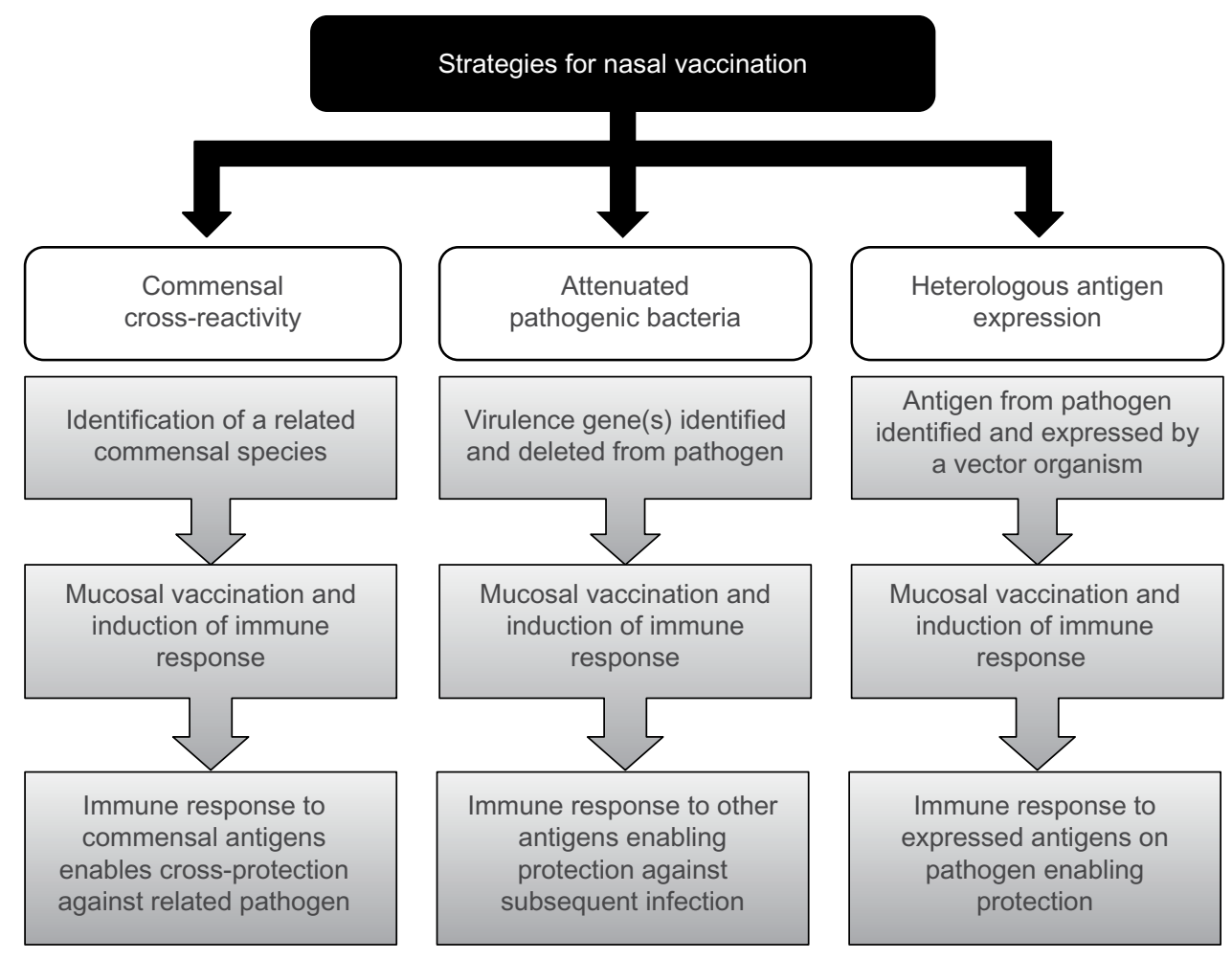

Figure I Summary of the principles employed in nasal vaccination strategies.

serum bactericidal effect. Furthermore, experimental $N$. lactamica colonization did not protect against subsequent natural $N$. meningitidis carriage acquisition. ${ }^{104}$ In fact, there has been some evidence to suggest that $N$. lactamica even protects $N$. meningitidis during colonization by triggering antibody-independent responses that do not induce a memory response. ${ }^{106}$ Nonetheless, the immunological response to colonization with $N$. lactamica could potentially protect against systemic infection with $N$. meningitidis, and further investigation of this strategy is ongoing. A similar strategy, in theory, could be applicable to other related pairs of pathogenic and nonpathogenic species, such as $S$. pneumoniae and $S$. mitis.

\section{Attenuated pathogenic bacteria}

Observations that natural mucosal exposure induces antibody and cellular immune responses to a range of bacterial antigens suggest that an alternative to current vaccine strategies could be the colonization of the nasopharynx with whole bacteria. This would reflect a more natural situation than subunit vaccines. To avoid the potential for causing active invasive infection, vaccination by artificial colonization of the nasopharynx would need to use attenuated strains unable to cause serious infection. This can now be achieved by targeted mutation of important virulence determinants, although these mutations could reduce the antigenicity of the attenuated strain. A critical aspect in the design of a live attenuated mucosal vaccine is achieving the balance between virulence attenuation for safety while retaining immunogenicity.

The use of attenuated $S$. pneumoniae as vaccines has been explored by several groups in animal models. In one example, genes encoding the capsule, Ply, and PspA were deleted rendering these strains avirulent yet still able to colonize and induce both systemic and mucosal antibodies that protected against disease in mice. ${ }^{107} \mathrm{~A}$ similar approach was the SPY1 mutant strain, where the capsule, teichoic acids, and Ply were deleted from a D39 S. pneumoniae strain and used for intranasal immunization. This protected against colonization and invasive disease caused by heterologous strains of $S$. pneumoniae in a T-cell- and B-cell-dependent manner. ${ }^{108,109}$ Further examples include the deletion of pep 27 leading to an avirulent strain with reduced capsule expression which when used to immunize mice intranasally protected against colonization and systemic infection ${ }^{110}$ and double mutation of the signal recognition pathway component $f t s Y$ and the calcium/magnesium transporter caxP/mgtA which when administered to the nasopharynx induces heterologous protection against OM, pneumonia, and invasive disease in a CD4+ cell-dependent manner. ${ }^{111}$ Another strategy for generating live vaccines is by creating auxotrophic organisms. In this way, key protective surface antigens such as PspA and the polysaccharide capsule for $S$. pneumoniae can be retained. For example, the deletion of the $p a b B$ gene 
creates an $S$. pneumoniae mutant strain auxotrophic for para-aminobenzoic acid and unable to replicate in the mammalian host. Systemic vaccination with this mutant was able to protect from homologous challenge in mouse models of sepsis and pneumonia. ${ }^{12}$ The attenuated mutant approach has been investigated for other bacteria. For example, in $S$. pyogenes, the deletion of the SpeB protease creates a mutant that is impaired in tRNA modification and has the potential to be used in vaccination strategies. ${ }^{113}$ Mutation of gidA in Salmonella enterica serovar Typhimurium also lends itself to a novel vaccine strategy for this bacterium. ${ }^{114}$

A potentially significant problem is that mouse and human data indicate that a reduced duration of nasopharyngeal colonization by $S$. pneumoniae significantly weakens the induced adaptive immune response. ${ }^{82,111,115}$ In mice, attenuated strains often have a reduced duration of colonization compared to wild-type bacteria, ${ }^{107,111,115}$ and this may therefore affect the efficacy of using attenuated mutants for preventing S. pneumoniae infections. For example, the $p a b B$ deletion strain is very rapidly cleared from the mouse nasopharynx and was only weakly immunogenic after nasopharyngeal administration. ${ }^{112}$ Repeated dosing of poorly colonizing strains may overcome this issue. ${ }^{82,107,110,115}$

Another potential problem is the surprising lack of cross-protection induced by attenuated strains in some of these studies. For example, although the protein antigens targeted by protective responses are largely conserved between $S$. pneumoniae strains, adaptive responses to one episode of colonization or systemic vaccination with a live attenuated vaccine either were not or only weakly cross-protective against heterologous strains in mouse models. ${ }^{12,115}$ The reasons for this poor cross-protective immunity are not clear and require further investigation to ensure that nasopharyngeal administration with a single strain can provide the broad heterologous protection required for an effective vaccine.

Reversion to wild type with loss of the attenuating mutation is a significant safety risk in the use of live attenuated bacteria as vaccines. This is a particular concern for pathogens such as $S$. pneumoniae that are naturally competent and are known to undergo recombination events during colonization. To avoid this, attenuated strains would need to contain at least two independent mutations. Another strategy to mitigate this risk would be to delete the competence machinery, rendering the strain unable to uptake foreign DNA and thereby preventing recombination events with the resident nasal flora which may possess similar virulence factors. In addition, the effect of administration of genetically modified bacteria to the nasopharynx on the existing nasal flora is not known, and will need to be evaluated carefully to ensure there are no unforeseen deleterious consequences.

\section{Heterologous expression of protective antigens}

An alternative approach has been to express recombinant protein in nonpathogenic species. Such strategies provide effective protection at the mucosal surface and during invasive disease. ${ }^{116}$ Lactic acid bacteria (LAB) are commonly used to manufacture foodstuffs and are therefore a safe alternative which are also known to elicit systemic and mucosal responses. ${ }^{117}$ The LAB, Lactobacillus casei, has been developed as an intranasal vaccine which expresses the $S$. pneumoniae protein antigen PspA and induces antibodies that protect mice from a systemic challenge. ${ }^{118}$ Another LAB, Lactococcus lactis, has also been used to express $S$. pneumoniae protein antigens including PspA, PpmA, PsaA, PppA, and SlrA ${ }^{119}$ or serotype $3^{120}$ and serotype $14^{121}$ capsular polysaccharides. Colonization with the L. lactis strains expressing $S$. pneumoniae capsules led to the induction of specific IgG and IgM antibodies. ${ }^{120,121}$ The oral commensal species Streptococcus gordonii, which also stimulates mucosal immunity, ${ }^{122}$ has been engineered to express protective antigens from $S$. pyogenes which are immunogenic in mouse models when inoculated intranasally and orally. ${ }^{123} \mathrm{~S}$. gordonii was also investigated as a means to express $N$. meningitidis antigens which induced bactericidal antibodies in intranasally immunized mice ${ }^{124}$ and Bordetella pertussis antigens which when used in oral colonization induced systemic and mucosal antibodies. ${ }^{122,125}$ These animal models and early studies in humans indicate that $S$. gordonii is a suitable vector for presenting heterologous antigens for a colonization approach vaccination strategy. ${ }^{126}$ However, a limitation of this strategy is the use of a limited number of antigens, which could restrict the range and strength of any protective immune response. Nevertheless, these early results indicate that this may be an area of potential future development applicable to a number of bacteria species, perhaps in combination with the use of closely related nonpathogenic species discussed earlier. For example, the expression of important $N$. meningitidis antigens in $N$. lactamica could increase the strength of cross-protective immunity induced by colonization with the modified $N$. lactamica strain.

\section{Overview and future directions}

Colonization of the nasopharynx is central to disease development and adaptive immune responses to potentially pathogenic organisms. Modulation of host-pathogen interac- 
tions at this site could be a powerful method of preventing serious bacterial infections for a range of common pathogens. There are several important characteristics which an attenuated microorganism must have to serve as a potential live human vaccine: 1) mutations must be stable and severely attenuate virulence to prevent the strain from causing lung or systemic infection; 2) two or more virulence genes should be mutated to minimize the chance of revertants developing; 3 ) the attenuated strain should retain the ability to stimulate significant increases in adaptive immune responses after nasopharyngeal administration, including mucosal immune responses that prevent lung infection; and 4) adaptive immunity to the mutant strain should result in cross-strain protection. Currently, most data showing the utility of nasopharyngeal colonization with attenuated or nonpathogenic organisms as a vaccination strategy have been obtained using animal models. However, the development of human models of nasopharyngeal carriage ${ }^{104,127-129}$ now allows the strategies using colonization to prevent infection to be tested in humans and to assess whether their potential can be fulfilled.

\section{Disclosure}

The authors report no conflicts of interest in this work.

\section{References}

1. Watson K, Carville K, Bowman J, et al. Upper respiratory tract bacterial carriage in Aboriginal and non-Aboriginal children in a semi-arid area of Western Australia. Pediatr Infect Dis J. 2006;25(9):782-790.

2. Kadioglu A, Weiser JN, Paton JC, Andrew PW. The role of Streptococcus pneumoniae virulence factors in host respiratory colonization and disease. Nat Rev Microbiol. 2008;6(4):288-301.

3. Simell B, Auranen K, Käyhty H, et al; Pneumococcal Carriage Group. The fundamental link between pneumococcal carriage and disease. Expert Rev Vaccines. 2012;11(7):841-855.

4. Blaser MJ, Falkow S. What are the consequences of the disappearing human microbiota? Nat Rev Microbiol. 2009;7(12):887-894.

5. Regev-Yochay G, Dagan R, Raz M, et al. Association between carriage of Streptococcus pneumoniae and Staphylococcus aureus in Children. JAMA. 2004;292(6):716-720.

6. Bogaert D, De Groot R, Hermans PWM. Streptococcus pneumoniae colonisation: the key to pneumococcal disease. Lancet Infect Dis. 2004;4(3):144-154.

7. Bisgaard H, Hermansen MN, Buchvald F, et al. Childhood asthma after bacterial colonization of the airway in neonates. $N$ Engl J Med. 2007;357(15):1487-1495.

8. Hilty M, Burke C, Pedro H, et al. Disordered microbial communities in asthmatic airways. PLoS One. 2010;5(1):e8578.

9. Marri PR, Stern DA, Wright AL, Billheimer D, Martinez FD. Asthmaassociated differences in microbial composition of induced sputum. J Allergy Clin Immunol. 2013;131(2): 346-52.e1-3.

10. Erb-Downward JR, Thompson DL, Han MK, et al. Analysis of the lung microbiome in the "healthy" smoker and in COPD. PLoS One. 2011;6(2):e16384.

11. Sze MA, Dimitriu PA, Hayashi S, et al. The lung tissue microbiome in chronic obstructive pulmonary disease. Am J Respir Crit Care Med. 2012;185(10):1073-1080.
12. Herbst T, Sichelstiel A, Schär C, et al. Dysregulation of allergic airway inflammation in the absence of microbial colonization. Am J Respir Crit Care Med. 2011;184(2):198-205.

13. Hagner S, Harb H, Zhao M, et al. Farm-derived Gram-positive bacterium Staphylococcus sciuri W620 prevents asthma phenotype in HDM- and OVA-exposed mice. Allergy. 2013;68(3):322-329.

14. Cahenzli J, Köller Y, Wyss M, Geuking MB, McCoy KD. Intestinal microbial diversity during early-life colonization shapes long-term IgE levels. Cell Host Microbe. 2013;14(5):559-570.

15. Richards L, Ferreira DM, Miyaji EN, Andrew PW, Kadioglu A. The immunising effect of pneumococcal nasopharyngeal colonisation; protection against future colonisation and fatal invasive disease. Immunobiology. 2010;215(4):251-263.

16. Cohen JM, Khandavilli S, Camberlein E, Hyams C, Baxendale HE, Brown JS. Protective contributions against invasive Streptococcus pneumoniae pneumonia of antibody and Th17-cell responses to nasopharyngeal colonisation. PLoS One. 2011;6(10):e25558.

17. Cohen JM, Chimalapati S, de Vogel C, van Belkum A, Baxendale HE, Brown JS. Contributions of capsule, lipoproteins and duration of colonisation towards the protective immunity of prior Streptococcus pneumoniae nasopharyngeal colonisation. Vaccine. 2012;30(30):4453-4459.

18. Zhang Z, Clarke TB, Weiser JN. Cellular effectors mediating Th17dependent clearance of pneumococcal colonization in mice. J Clin Invest. 2009;119(7):1899-1909.

19. Wilson R, Cohen JM, José RJ, de Vogel C, Baxendale H, Brown JS. Protection against Streptococcus pneumoniae lung infection after nasopharyngeal colonization requires both humoral and cellular immune responses. Mucosal Immunol. 2015;8(3):627-639.

20. Wright AKA, Bangert M, Gritzfeld JF, et al. Experimental human pneumococcal carriage augments IL-17A-dependent T-cell defence of the lung. PLoS Pathog. 2013;9(3):e1003274.

21. Ferreira DM, Neill DR, Bangert M, et al. Controlled human infection and rechallenge with Streptococcus pneumoniae reveals the protective efficacy of carriage in healthy adults. Am J Respir Crit Care Med. 2013;187(8):855-864.

22. Gray BM, Dillon HC Jr. Epidemiological studies of Streptococcus pneumoniae in infants: antibody to types 3, 6, 14, and 23 in the first two years of life. J Infect Dis. 1988;158(5):948-955.

23. Loda FA, Collier AM, Glezen WP, Strangert K, Clyde WA Jr, Denny FW. Occurrence of Diplococcus pneumoniae in the upper respiratory tract of children. J Pediatr. 1975;87(6 pt 2):1087-1093.

24. Syrjänen RK, Kilpi TM, Kaijalainen TH, Herva EE, Takala AK. Nasopharyngeal carriage of Streptococcus pneumoniae in Finnish children younger than 2 years old. J Infect Dis. 2001;184(4):451-459.

25. Regev-Yochay G, Raz M, Dagan R, et al. Nasopharyngeal carriage of Streptococcus pneumoniae by adults and children in community and family settings. Clin Infect Dis. 2004;38(5):632-639.

26. Goldblatt D, Hussain M, Andrews N, et al. Antibody responses to nasopharyngeal carriage of Streptococcus pneumoniae in adults: a longitudinal household study. J Infect Dis. 2005;192(3):387-393.

27. Sá-Leão R, Nunes S, Brito-Avô A, et al. High rates of transmission of and colonization by Streptococcus pneumoniae and Haemophilus influenzae within a day care center revealed in a longitudinal study. J Clin Microbiol. 2008;46(1):225-234.

28. Gray BM, Converse GM, Dillon HC Jr. Epidemiologic studies of Streptococcus pneumoniae in infants: acquisition, carriage, and infection during the first 24 months of life. J Infect Dis. 1980;142(6): 923-933.

29. Abdullahi O, Karani A, Tigoi CC, et al. Rates of acquisition and clearance of pneumococcal serotypes in the nasopharynges of children in Kilifi District, Kenya. J Infect Dis. 2012;206(7):1020-1029.

30. Turner P, Turner C, Jankhot A, et al. A longitudinal study of Streptococcus pneumoniae carriage in a cohort of infants and their mothers on the Thailand-Myanmar border. PLoS One. 2012;7(5):e38271. 
31. Kandasamy R, Gurung M, Thapa A, et al. Multi-serotype pneumococcal nasopharyngeal carriage prevalence in vaccine naïve Nepalese children, assessed using molecular serotyping. PLoS One. 2015; 10(2): 0114286.

32. Kamng'ona AW, Hinds J, Bar-Zeev N, et al. High multiple carriage and emergence of Streptococcus pneumoniae vaccine serotype variants in Malawian children. BMC Infect Dis. 2015;15:234.

33. Ekdahl K, Ahlinder I, Hansson HB, et al. Duration of nasopharyngeal carriage of penicillin-resistant Streptococcus pneumoniae: experiences from the South Swedish Pneumococcal Intervention Project. Clin Infect Dis. 1997;25(5):1113-1117.

34. Sleeman KL, Griffiths D, Shackley F, et al. Capsular serotype-specific attack rates and duration of carriage of Streptococcus pneumoniae in a population of children. J Infect Dis. 2006;194(5):682-688.

35. O'Brien KL, Wolfson LJ, Watt JP, et al. Burden of disease caused by Streptococcus pneumoniae in children younger than 5 years: global estimates. Lancet. 2009;374(9693):893-902.

36. Black RE, Cousens S, Johnson HL, et al. Global, regional, and national causes of child mortality in 2008: a systematic analysis. Lancet 2010;375(9730):1969-1987.

37. Walker CLF, Rudan I, Liu L, et al. Global burden of childhood pneumonia and diarrhoea. Lancet. 2013;381(9875):1405-1416.

38. Isaacman DJ, McIntosh ED, Reinert RR. Burden of invasive pneumococcal disease and serotype distribution among Streptococcus pneumoniae isolates in young children in Europe: impact of the 7 -valent pneumococcal conjugate vaccine and considerations for future conjugate vaccines. Int J Infect Dis. 2010;14(3):e197-e209.

39. Davis SM, Deloria-Knoll M, Kassa HT, O'Brien KL. Impact of pneumococcal conjugate vaccines on nasopharyngeal carriage and invasive disease among unvaccinated people: review of evidence on indirect effects. Vaccine. 2013;32(1):133-145.

40. Moberley S, Holden J, Tatham DP, Andrews RM. Vaccines for preventing pneumococcal infection in adults. Cochrane Database Syst Rev. 2013;1:CD000422.

41. Yildirim I, Hanage WP, Lipsitch M, et al. Serotype specific invasive capacity and persistent reduction in invasive pneumococcal disease. Vaccine. 2010;29(2):283-288.

42. Hanage WP, Finkelstein JA, Huang SS, et al. Evidence that pneumococcal serotype replacement in Massachusetts following conjugate vaccination is now complete. Epidemics. 2010;2(2):80-84.

43. Flasche S, Van Hoek AJ, Sheasby E, et al. Effect of pneumococcal conjugate vaccination on serotype-specific carriage and invasive disease in England: a cross-sectional study. PLoS Med. 2011;8(4):e1001017.

44. Biesbroek G, Wang X, Keijser BJF, et al. Seven-valent pneumococcal conjugate vaccine and nasopharyngeal microbiota in healthy children. Emerg Infect Dis. 2014;20(2):201-210.

45. Spijkerman J, Prevaes SMPJ, van Gils EJM, et al. Long-term effects of pneumococcal conjugate vaccine on nasopharyngeal carriage of $S$. pneumoniae, S. aureus, H. influenzae and M. catarrhalis. PLoS One. 2012; 7(6):e39730.

46. Pericone CD, Overweg K, Hermans PW, Weiser JN. Inhibitory and bactericidal effects of hydrogen peroxide production by Streptococcus pneumoniae on other inhabitants of the upper respiratory tract. Infect Immun. 2000;68(7):3990-3997.

47. Johnson AP. The pathogenic potential of commensal species of Neisseria. J Clin Pathol. 1983;36(2):213-223.

48. Stephens DS. Uncloaking the meningococcus: dynamics of carriage and disease. Lancet. 1999;353(9157):941-942.

49. Cartwright KAV, Stuart JM, Jones DM, Noah ND. The Stonehouse survey: nasopharyngeal carriage of meningococci and Neisseria lactamica. Epidemiol Infect. 1987;99(3):591-601.

50. Caugant DA, Høiby EA, Magnus P, et al. Asymptomatic carriage of Neisseria meningitidis in a randomly sampled population. $J$ Clin Microbiol. 1994;32(2):323-330.

51. Davies AL, O'Flanagan D, Salmon RL, Coleman TJ. Risk factors for Neisseria meningitides carriage in a school during a community outbreak of meningococcal infection. Epidemiol Infect. 1996;117(2):259.
52. Boisier P, Nicolas P, Djibo S, et al. Meningococcal meningitis: unprecedented incidence of serogroup X-related cases in 2006 in Niger. Clin Infect Dis. 2007;44(5):657-663.

53. Trotter CL, Maiden MCJ. Meningococcal vaccines and herd immunity: lessons learned from serogroup $\mathrm{C}$ conjugate vaccination programs. Expert Rev Vaccines. 2009;8(7):851-861.

54. Hepkema H, Pouwels KB, van der Ende A, Westra TA, Postma MJ. Meningococcal serogroup $\mathrm{A}, \mathrm{C}, \mathrm{W}_{123}$ and $\mathrm{Y}$ conjugated vaccine: a cost-effectiveness analysis in the Netherlands. PLoS One. 2013; 8(5):e65036.

55. Könönen E. Anaerobes in the upper respiratory tract in infancy. Anaerobe. 2005;11(3):131-136.

56. Bogaert D, Keijser B, Huse S, et al. Variability and diversity of nasopharyngeal microbiota in children: a metagenomic analysis. PLoS One. 2011;6(2):e17035.

57. Hilty M, Qi W, Brugger SD, et al. Nasopharyngeal microbiota in infants with acute otitis media. J Infect Dis. 2012;205(7):1048-1055.

58. van der Waaij D, Berghuis-de Vries JM. Lekkerkerk Lekkerkerk-v. Colonization resistance of the digestive tract in conventional and antibiotic-treated mice. J Hyg (Lond). 1971;69(3):405-411.

59. Kamada N, Chen GY, Inohara N, Núñez G. Control of pathogens and pathobionts by the gut microbiota. Nat Immunol. 2013;14(7):685-690.

60. Wescombe PA, Heng NCK, Burton JP, Chilcott CN, Tagg JR. Streptococcal bacteriocins and the case for Streptococcus salivarius as model oral probiotics. Future Microbiol. 2009;4(7):819-835.

61. Santagati M, Scillato M, Patanè F, Aiello C, Stefani S. Bacteriocinproducing oral streptococci and inhibition of respiratory pathogens. FEMS Immunol Med Microbiol. 2012;65(1):23-31.

62. Hammami R, Fernandez B, Lacroix C, Fliss I. Anti-infective properties of bacteriocins: an update. Cell Mol Life Sci. 2013;70(16):2947-2967.

63. Iwase T, Uehara Y, Shinji H, et al. Staphylococcus epidermidis Esp inhibits Staphylococcus aureus biofilm formation and nasal colonization. Nature. 2010;465(7296):346-349.

64. Regev-Yochay G, Trzcinski K, Thompson CM, Lipsitch M, Malley R. $\mathrm{SpxB}$ is a suicide gene of Streptococcus pneumoniae and confers a selective advantage in an in vivo competitive colonization model. J Bacteriol. 2007;189(18):6532-6539.

65. Shakhnovich EA, King SJ, Weiser JN. Neuraminidase expressed by Streptococcus pneumoniae desialylates the lipopolysaccharide of Neisseria meningitidis and Haemophilus influenzae: a paradigm for interbacterial competition among pathogens of the human respiratory tract. Infect Immun. 2002;70(12):7161-7164.

66. Crowe CC, Sanders WE, Longley S. Bacterial interference. II. Role of the normal throat flora in prevention of colonization by group A Streptococcus. J Infect Dis. 1973;128(4):527-532.

67. Lysenko ES, Ratner AJ, Nelson AL, Weiser JN. The role of innate immune responses in the outcome of interspecies competition for colonization of mucosal surfaces. PLoS Pathog. 2005;1(1):e1.

68. Turner P, Turner C, Green N, et al. Serum antibody responses to pneumococcal colonization in the first 2 years of life: results from an SE Asian longitudinal cohort study. Clin Microbiol Infect. 2013; 19(12):E551-E558.

69. Soininen A, Pursiainen H, Kilpi T, Käyhty H. Natural development of antibodies to pneumococcal capsular polysaccharides depends on the serotype: association with pneumococcal carriage and acute otitis media in young children. $J$ Infect Dis. 2001;184(5):569-576.

70. Musher DM, Groover JE, Reichler MR, et al. Emergence of antibody to capsular polysaccharides of Streptococcus pneumoniae during outbreaks of pneumonia: association with nasopharyngeal colonization. Clin Infect Dis. 1997;24(3):441-446.

71. Salt $\mathrm{P}, \mathrm{Banner} \mathrm{C}, \mathrm{Oh} \mathrm{S}$, et al. Social mixing with other children during infancy enhances antibody response to a pneumococcal conjugate vaccine in early childhood. Clin Vaccine Immunol. 2007;14(5):593-599.

72. Lebon A, Verkaik NJ, Labout JAM, et al. Natural antibodies against several pneumococcal virulence proteins in children during the prepneumococcal-vaccine era: the generation R study. Infect Immun. 2011; 79(4):1680-1687. 
73. Rapola S, Jäntti V, Haikala R, et al. Natural development of antibodies to pneumococcal surface protein A, pneumococcal surface adhesin A, and pneumolysin in relation to pneumococcal carriage and acute otitis media. J Infect Dis. 2000;182(4):1146-1152.

74. Simell B, Ahokas P, Lahdenkari M, et al. Pneumococcal carriage and acute otitis media induce serum antibodies to pneumococcal surface proteins CbpA and PhtD in children. Vaccine. 2009;27(34):4615-4621.

75. Prevaes SMPJ, van Wamel WJB, de Vogel CP, et al. Nasopharyngeal colonization elicits antibody responses to staphylococcal and pneumococcal proteins that are not associated with a reduced risk of subsequent carriage. Infect Immun. 2012;80(6):2186-2193.

76. McCool TL, Cate TR, Moy G, Weiser JN. The immune response to pneumococcal proteins during experimental human carriage. J Exp Med. 2002;195(3):359-365.

77. Jefferies JMC, Johnston CHG, Kirkham L-AS, et al. Presence of nonhemolytic pneumolysin in serotypes of Streptococcus pneumoniae associated with disease outbreaks. J Infect Dis. 2007;196(6): 936-944.

78. Lu YJ, Gross J, Bogaert D, et al. Interleukin-17A mediates acquired immunity to pneumococcal colonization. PLoS Pathog. 2008;4(9): e1000159.

79. Roche A, Richard AL, Rahkola JT, Janoff EN, Weiser JN. Antibody blocks acquisition of bacterial colonization through agglutination. Mucosal Immunol. 2015;8(1):176-185.

80. Park H-S, Francis KP, Yu J, Cleary PP. Membranous cells in nasalassociated lymphoid tissue: a portal of entry for the respiratory mucosal pathogen group A Streptococcus. J Immunol. 2003;171(5):2532-2537.

81. Wang B, Dileepan T, Briscoe S, et al. Induction of TGF-beta1 and TGF-beta1-dependent predominant Th17 differentiation by group A streptococcal infection. Proc Natl Acad Sci U S A. 2010;107(13): 5937-5942.

82. Wright AKA, Ferreira DM, Gritzfeld JF, et al. Human nasal challenge with Streptococcus pneumoniae is immunising in the absence of carriage. PLoS Pathog. 2012;8(4):e1002622.

83. Kremastinou J, Tzanakaki G, Pagalis A, Theodondou M, Weir DM, Blackwell CC. Detection of IgG and IgM to meningococcal outer membrane proteins in relation to carriage of Neisseria meningitidis or Neisseria lactamica. FEMS Immunol Med Microbiol. 1999;24(1):73-78.

84. Jones GR, Christodoulides M, Brooks JL, Miller ARO, Cartwright KAV, Heckels JE. Dynamics of carriage of Neisseria meningitidis in a group of military recruits: subtype stability and specificity of the immune response following colonization. J Infect Dis. 1998;178(2):451-459.

85. Robinson K, Neal KR, Howard C. Characterization of humoral and cellular immune responses elicited by meningococcal carriage. Infect Immun. 2002;70(3):1301-1309.

86. Sánchez S, Troncoso G, Ferreirós C, Criado M. Evaluation of crossreactive antigens as determinants of cross-bactericidal activity in pathogenic and commensal Neisseria. Vaccine. 2001;19(25-26):3390-3398.

87. Sánchez S, Troncoso G, Criado MT, Ferreirós C. In vitro induction of memory-driven responses against Neisseria meningitidis by priming with Neisseria lactamica. Vaccine. 2002;20(23-24):2957-2963.

88. Frey SE, Lottenbach KR, Hill H, et al. A Phase I, dose-escalation trial in adults of three recombinant attenuated Salmonella Typhi vaccine vectors producing Streptococcus pneumoniae surface protein antigen PspA. Vaccine. 2013;31(42):4874-4880.

89. Tennant SM, Levine MM. Live attenuated vaccines for invasive Salmonella infections. Vaccine. 2015;33(suppl 3):C36-C41.

90. Alvarez-Olmos MI, Oberhelman RA. Probiotic agents and infectious diseases: a modern perspective on a traditional therapy. Clin Infect Dis. 2001;32(11):1567-1576.

91. Niittynen L, Pitkäranta A, Korpela R. Probiotics and otitis media in children. Int J Pediatr Otorhinolaryngol. 2012;76(4):465-470.

92. Glück U, Gebbers J-O. Ingested probiotics reduce nasal colonization with pathogenic bacteria (Staphylococcus aureus, Streptococcus pneumoniae, and beta-hemolytic streptococci). Am J Clin Nutr. 2003;77(2):517-520.
93. Hatakka K, Savilahti E, Pönkä A, et al. Effect of long term consumption of probiotic milk on infections in children attending day care centres: double blind, randomised trial. BMJ. 2001;322(7298):1327.

94. Hatakka K, Blomgren K, Pohjavuori S, et al. Treatment of acute otitis media with probiotics in otitis-prone children-a double-blind, placebocontrolled randomised study. Clin Nutr. 2007;26(3):314-321.

95. Bernstein JM, Sagahtaheri-Altaie S, Dryja DM, Wactawski-Wende J. Bacterial interference in nasopharyngeal bacterial flora of otitisprone and non-otitis-prone children. Acta Otorhinolaryngol Belg. 1994;48(1):1-9.

96. Bernstein JM, Haase E, Hasse E, et al. Bacterial interference of penicillin-sensitive and -resistant Streptococcus pneumoniae by Streptococcus oralis in an adenoid organ culture: implications for the treatment of recurrent upper respiratory tract infections in children and adults. Ann Otol Rhinol Laryngol. 2006;115(5):350-356.

97. Di Pierro F, Donato G, Fomia F, et al. Preliminary pediatric clinical evaluation of the oral probiotic Streptococcus salivarius K12 in preventing recurrent pharyngitis and/or tonsillitis caused by Streptococcus pyogenes and recurrent acute otitis media. Int J Gen Med. 2012;5:991-997.

98. Di Pierro F, Adami T, Rapacioli G, Giardini N, Streitberger C. Clinical evaluation of the oral probiotic Streptococcus salivarius K12 in the prevention of recurrent pharyngitis and/or tonsillitis caused by Streptococcus pyogenes in adults. Expert Opin Biol Ther. 2013;13(3):339-343.

99. Villena J, Barbieri N, Salva S, Herrera M, Alvarez S. Enhanced immune response to pneumococcal infection in malnourished mice nasally treated with heat-killed Lactobacillus casei. Microbiol Immunol. 2009;53(11):636-646

100. Salva S, Villena J, Alvarez S. Immunomodulatory activity of Lactobacillus rhamnosus strains isolated from goat milk: impact on intestinal and respiratory infections. Int J Food Microbiol. 2010;141(1-2):82-89.

101. Roos K, Håkansson EG, Holm S. Effect of recolonisation with "interfering" alpha streptococci on recurrences of acute and secretory otitis media in children: randomised placebo controlled trial. $B M J$. 2001;322(7280):210-212.

102. Tano K, Grahn Håkansson E, Holm SE, Hellström S. A nasal spray with alpha-haemolytic streptococci as long term prophylaxis against recurrent otitis media. Int J Pediatr Otorhinolaryngol. 2002;62(1):17-23.

103. Troncoso G, Sánchez S, Moreda M, Criado MT, Ferreirós CM. Antigenic cross-reactivity between outer membrane proteins of Neisseria meningitidis and commensal Neisseria species. FEMS Immunol Med Microbiol. 2000;27(2):103-109.

104. Evans CM, Pratt CB, Matheson M, et al. Nasopharyngeal colonization by Neisseria lactamica and induction of protective immunity against Neisseria meningitidis. Clin Infect Dis. 2011;52(1):70-77.

105. Sánchez S, Abel A, Arenas J, Criado MT, Ferreirós CM. Cross-linking analysis of antigenic outer membrane protein complexes of Neisseria meningitidis. Res Microbiol. 2006;157(2):136-142.

106. Vaughan AT, Gorringe A, Davenport V, Williams NA, Heyderman RS. Absence of mucosal immunity in the human upper respiratory tract to the commensal bacteria Neisseria lactamica but not pathogenic Neisseria meningitidis during the peak age of nasopharyngeal carriage. J Immunol. 2009;182(4):2231-2240.

107. Roche AM, King SJ, Weiser JN. Live attenuated Streptococcus pneumoniae strains induce serotype-independent mucosal and systemic protection in mice. Infect Immun. 2007;75(5):2469-2475.

108. Wu K, Yao R, Wang H, et al. Mucosal and systemic immunization with a novel attenuated pneumococcal vaccine candidate confer serotype independent protection against Streptococcus pneumoniae in mice. Vaccine. 2014;32(33):4179-4188.

109. Xu X, Wang H, Liu Y, et al. Mucosal immunization with the live attenuated vaccine SPY1 induces humoral and Th2-Th17-regulatory T cell cellular immunity and protects against pneumococcal infection. Infect Immun. 2015;83(1):90-100.

110. Kim E-HH, Choi S-YY, Kwon M-KK, et al. Streptococcus pneumoniae pep27 mutant as a live vaccine for serotype-independent protection in mice. Vaccine. 2012;30(11):2008-2019. 
111. Rosch JW, Iverson AR, Humann J, et al. A live-attenuated pneumococcal vaccine elicits CD4+T-cell dependent class switching and provides serotype independent protection against acute otitis media. EMBO Mol Med. 2014;6(1):141-154.

112. Chimalapati S, Cohen J, Camberlein E, et al. Infection with conditionally virulent Streptococcus pneumoniae $\Delta$ pab strains induces antibody to conserved protein antigens but does not protect against systemic infection with heterologous strains. Infect Immun. 2011;79(12):4965-4976.

113. Cho KH, Caparon MG. tRNA modification by GidA/MnmE is necessary for Streptococcus pyogenes virulence: a new strategy to make live attenuated strains. Infect Immun. 2008;76(7):3176-3186.

114. Shippy DC, Fadl AA. Immunological characterization of a gidA mutant strain of Salmonella for potential use in a live-attenuated vaccine. BMC Microbiol. 2012;12:286.

115. Cohen JM, Wilson R, Shah P, Baxendale HE, Brown JS. Lack of cross-protection against invasive pneumonia caused by heterologous strains following murine Streptococcus pneumoniae nasopharyngeal colonisation despite whole cell ELISAs showing significant crossreactive IgG. Vaccine. 2013;31(19):2328-2332.

116. Hanniffy SB, Carter AT, Hitchin E, Wells JM. Mucosal delivery of a pneumococcal vaccine using Lactococcus lactis affords protection against respiratory infection. J Infect Dis. 2007;195(2):185-193.

117. Wells JM, Mercenier A. Mucosal delivery of therapeutic and prophylactic molecules using lactic acid bacteria. Nat Rev Microbiol. 2008;6(5): 349-362.

118. Campos IB, Darrieux M, Ferreira DM, et al. Nasal immunization of mice with Lactobacillus casei expressing the Pneumococcal Surface Protein A: induction of antibodies, complement deposition and partial protection against Streptococcus pneumoniae challenge. Microbes Infect. 2008;10(5):481-488.

119. Medina MS, Vintiñi EO, Villena J, Raya RR, Alvarez SG. Lactococcus lactis as an adjuvant and delivery vehicle of antigens against pneumococcal respiratory infections. Bioeng Bugs. 2014;1(5):313-325.

120. Gilbert C, Robinson K, Le Page RW, Wells JM. Heterologous expression of an immunogenic pneumococcal type 3 capsular polysaccharide in Lactococcus lactis. Infect Immun. 2000;68(6):3251-3260.
121. Nierop Groot MN, Godefrooij J, Kleerebezem M. Heterologous expression of the pneumococcal serotype 14 polysaccharide in Lactococcus lactis requires lactococcal epsABC regulatory genes. Appl Environ Microbiol. 2008;74(3):912-915.

122. Lee SF, Halperin SA, Wang H, MacArthur A. Oral colonization and immune responses to Streptococcus gordonii expressing a pertussis toxin S1 fragment in mice. FEMS Microbiol Lett. 2002;208(2) 175-178.

123. Medaglini D, Pozzi G, King TP, Fischetti VA. Mucosal and systemic immune responses to a recombinant protein expressed on the surface of the oral commensal bacterium Streptococcus gordonii after oral colonization. Proc Natl Acad Sci U S A. 1995;92(15):6868-6872.

124. Ciabattini A, Giomarelli B, Parigi R, et al. Intranasal immunization of mice with recombinant Streptococcus gordonii expressing NadA of Neisseria meningitidis induces systemic bactericidal antibodies and local IgA. Vaccine. 2008;26(33):4244-4250.

125. Lee SF, Halperin SA, Knight JB, Tait A. Purification and immunogenicity of a recombinant Bordetella pertussis S1S3FHA fusion protein expressed by Streptococcus gordonii. Appl Environ Microbiol. 2002;68(9):4253-4258.

126. Kotloff KL, Wasserman SS, Jones KF, et al. Clinical and microbiological responses of volunteers to combined intranasal and oral inoculation with a Streptococcus gordonii carrier strain intended for future use as a group A Streptococcus vaccine. Infect Immun. 2005;73(4) 2360-2366.

127. Ferreira DM, Jambo KC, Gordon SB. Experimental human pneumococcal carriage models for vaccine research. Trends Microbiol. 2011; 19(9):464-470.

128. Deasy AM, Guccione E, Dale AP, et al. Nasal inoculation of the commensal Neisseria lactamica inhibits carriage of Neisseria meningitidis by young adults: a controlled human infection study. Clin Infect Dis. 2015; 60(10):1512-1520.

129. Winokur PL, Chaloner K, Doern GV, Ferreira J, Apicella MA. Safety and immunological outcomes following human inoculation with nontypeable Haemophilus influenzae. J Infect Dis. 2013;208(5): $728-738$.
Vaccine: Development and Therapy

\section{Publish your work in this journal}

Vaccine: Development and Therapy is an international, peer-reviewed, open access journal that spans the spectrum of vaccine design and development through to clinical applications. The journal is characterized by the rapid reporting of application notes, reviews, original research and clinical studies in all therapeutic areas. Clinical outcomes, patient safety, and programs for

\section{Dovepress}

the development and effective, safe, and sustained use of vaccines will be a feature of the journal. The manuscript management system is completely online and includes a very quick and fair peer-review system. Visit http://www. dovepress.com/testimonials.php to read real quotes from published authors. 